\title{
PULSED DYE LASER LITHOTRIPSY OF BILE DUCT STONES
}

\author{
T. PONCHON, P. GAGNON, P.J. VALETTE, L. HENRY and A. CHAVAILLON \\ Department of Digestive Diseases, INSERM U281, Hôpital E. Herriot, Lyon, France
}

\begin{abstract}
Efficacy and safety of pulsed dye laser lithotripsy was tested in 28 consecutive patients in whom bile duct stones could not be extracted following endoscopic sphincterotomy.
\end{abstract}

Methods: The patients had 1 to 6 (mean:1.9) bile duct stones (diameter:10-35mm, mean:18mm) located in the common bile duct (20 cases), the intra-hepatic bile ducts (7), or in a long cystic duct stump (1). The same flashlamp pulsed dye laser was used for all patients (Pulsolith Technomed, wavelength: $504 \mathrm{~nm}$, pulse duration: $2,4 \mu s$, energy output: $60-90 \mathrm{~mJ} /$ pulse, pulse rate: $5 \mathrm{~Hz}$, maximal number of pulses per laser session: 3000 ). Different approaches were tested depending on the presence of a T-tube and on the localization of the bile duct stones. When a T-tube was already in place (7 cases), the lithotripsy was done under direct vision using a choledochoscope inserted through the T-tube tract. In 21 patients without a T-tube in place, the lithotripsy was done under fluoroscopy using a retrograde approach in case of common bile duct stones (16 cases) or under direct vision using a choledochoscope inserted through a percutaneous transhepatic tract in case of intrahepatic bile duct stones (5 cases). For the 16 cases done under fluoroscopy, either a balloon catheter (10 cases) or a basket $(6$ cases) was used in order to position the fibre in the middle of the biliary lumen and in contact with the stone.

Results: Fragmentation of all the bile duct stones and a complete bile duct clearance were obtained in the 12 cases done under direct vision as compared to only 5 of 16 cases done under fluoroscopic control. Moreover, 7 of the 11 failures using the latter approach were offered another session using a choledochoscope inserted through a percutaneous transhepatic tract and were also successfully treated. Failures under fluoroscopy were attributed to:1- inability to position the balloon catheter ( 2 cases), 2- inability to pass the choledocho-duodenal angle with the fibre (5), tangential instead of perpendicular positioning of the fibre with respect to the stone (3), fracture of the fibre unnoticed during the procedure (1). No complication related to the laser beam was noted.

cenclusion: Pulsed dye laser lithotripsy of bile duct stones unable to be removed by standard endoscopic techniques is safe and efficacious provided that it is performed under direct vision. Technical refinements are needed before it can be reliably done under fluoroscopy. 